\title{
EP300 Protects from Light-Induced Retinopathy in Zebrafish
}

\section{OPEN ACCESS}

Edited by:

Chiranjib Chakraborty

Galgotias University, India

Reviewed by:

Vincenzo Bramanti,

University of Catania, Italy

Pavel I. Nedvetsky,

Vlaams Instituut voor Biotechnologie,

Belgium

${ }^{*}$ Correspondence:

Toshio Tanaka

tanaka@doc.medic.mie-u.ac.jp

${ }^{\dagger}$ These authors have contributed equally to this work.

Specialty section:

This article was submitted to

Experimental Pharmacology and Drug

Discovery,

a section of the journal

Frontiers in Pharmacology

Received: 01 March 2016 Accepted: 05 May 2016

Published: 19 May 2016

Citation:

Kawase R, Nishimura Y, Ashikawa Y, Sasagawa S, Murakami S, Yuge M, Okabe S, Kawaguchi K, Yamamoto $H$,

Moriyuki K, Yamane S, Tsuruma K,

Shimazawa M, Hara H and Tanaka T

(2016) EP300 Protects from

Light-Induced Retinopathy in

Zebrafish. Front. Pharmacol. 7:126.

doi: 10.3389/fphar.2016.00126

\begin{abstract}
Reiko Kawase ${ }^{1 \dagger}$, Yuhei Nishimura ${ }^{1,2,3,4,5 t}$, Yoshifumi Ashikawa ${ }^{1}$, Shota Sasagawa ${ }^{1}$, Soichiro Murakami ${ }^{1}$, Mizuki Yuge ${ }^{1}$, Shiko Okabe ${ }^{1}, K_{\text {Ko Kawaguchi }}{ }^{1}$, Hiroshi Yamamoto ${ }^{6}$, Kazumi Moriyuki ${ }^{6}$, Shinsaku Yamane ${ }^{6}$, Kazuhiro Tsuruma ${ }^{7}$, Masamitsu Shimazawa ${ }^{7}$, Hideaki Hara ${ }^{7}$ and Toshio Tanaka ${ }^{1,2,3,4,5 *}$
\end{abstract}

${ }^{1}$ Department of Molecular and Cellular Pharmacology, Pharmacogenomics, and Pharmacoinformatics, Mie University Graduate School of Medicine, Tsu, Japan, ${ }^{2}$ Mie University Medical Zebrafish Research Center, Tsu, Japan, ${ }^{3}$ Department of Systems Pharmacology, Mie University Graduate School of Medicine, Tsu, Japan, ${ }^{4}$ Department of Omics Medicine, Mie University Industrial Technology Innovation Institute, Tsu, Japan, ${ }^{5}$ Department of Bioinformatics, Mie University Life Science Research Center, Tsu, Japan, ${ }^{6}$ Ono Pharmaceutical Co, Ltd. Osaka, Japan, ${ }^{7}$ Molecular Pharmacology, Department of Biofunctional Evaluation, Gifu Pharmaceutical University, Gifu, Japan

Exposure of rhodopsin to bright white light can induce photoreceptor cell damage and degeneration. However, a comprehensive understanding of the mechanisms underlying light-induced retinopathy remains elusive. In this study, we performed comparative transcriptome analysis of three rodent models of light-induced retinopathy, and we identified 37 genes that are dysregulated in all three models. Gene ontology analysis revealed that this gene set is significantly associated with a cytokine signaling axis composed of signal transducer and activator of transcription 1 and 3 (STAT1/3), interleukin 6 signal transducer (IL6ST), and oncostatin M receptor (OSMR). Furthermore, the analysis suggested that the histone acetyltransferase EP300 may be a key upstream regulator of the STAT1/3-IL6ST/OSMR axis. To examine the role of EP300 directly, we developed a larval zebrafish model of light-induced retinopathy. Using this model, we demonstrated that pharmacological inhibition of EP300 significantly increased retinal cell apoptosis, decreased photoreceptor cell outer segments, and increased proliferation of putative Müller cells upon exposure to intense light. These results suggest that EP300 may protect photoreceptor cells from light-induced damage and that activation of EP300 may be a novel therapeutic approach for the treatment of retinal degenerative diseases.

Keywords: light-induced retinopathy, EP300, STAT3, apoptosis, comparative transcriptome analysis, zebrafish, systems pharmacology

\section{INTRODUCTION}

Retinal photoreceptor cells are uniquely adapted to function over a wide range of ambient light conditions. However, in most species, prolonged exposure to intense visible light can lead to photoreceptor cell damage (reviewed in Wenzel et al., 2005; Organisciak and Vaughan, 2010; Chen et al., 2016). Light-induced retinal damage serves as a model to study human retinal degeneration arising from environmental insult, aging, and genetic disease. Accordingly, animal models of light-induced retinopathy have been used successfully to develop therapeutic approaches for retinal degenerative diseases (Tsuruma et al., 2014; Shimazawa et al., 2015). While many of the light-induced retinopathy models share similar mechanisms, there are also significant differences (reviewed in Organisciak and Vaughan, 2010). For example, inhibition of the transcription factor 
(TF) activator protein-1 protects against light damage in mice (Wenzel et al., 2001) but not in T4R rhodopsin mutant dogs (Gu et al., 2009). Similarly, exposure to intense white light causes degeneration of retinal pigment epithelial cells in rats but not in mice (Remé et al., 1998). These findings suggest that there may be shared and unique pathways of light-induced retinal cell death in the various models.

Comparative transcriptome analysis has been used successfully to identify common and divergent genomic responses in a disease entity caused by various etiologies in different species (Nishimura et al., 2007, 2015b; Oka et al., 2010). Here, we sought to identify genes that are dysregulated in multiple models of light-induced retinopathy and might therefore, be exploited as novel therapeutic targets. We compared the transcriptomes of three rodent models of lightinduced retinopathy (Rattner and Nathans, 2005; Natoli et al., 2010; Hadziahmetovic et al., 2012) and identified 37 genes that were differentially expressed in all three models. We also demonstrated that (i) a signaling axis composed of signal transducer and activator of transcription 1 and 3 (STAT1/3), interleukin 6 signal transducer (IL6ST), and oncostatin M receptor (STAT1/3-IL6ST/OSMR), which is involved in the neuroprotective role of IL-6 (Jung et al., 2011), plays a key role in light-induced retinopathy, (ii) the histone acetyltransferase (HAT) EP300 is a key upstream regulator of the STAT1/3IL6ST/OSMR axis, and (iii) EP300 plays a protective role in the larval zebrafish model of light-induced retinopathy, suggesting that EP300 may be a novel therapeutic target for retinal degenerative diseases.

\section{MATERIALS AND METHODS}

\section{Ethics Statement}

This study was carried out in strict accordance with Japanese law, including the Human Treatment and Management of Animals Act (2014), Standards Relating to the Care and Management of Laboratory Animals and Relief of Pain (2013), and the Guidelines for Proper Conduct of Animal Experiments (Science Council of Japan, 2006). All experiments were performed on animals under 2-phenoxyethanol anesthesia, and all efforts were made to minimize animal suffering.

\section{Compounds}

The HAT inhibitor C646 (Bowers et al., 2010), phenylthiourea, and 5-bromo-2-deoxyuridine (BrdU) were purchased from Sigma (St. Louis, MO, USA). Stock solutions of C646 and phenylthiourea were prepared in dimethyl sulfoxide (DMSO; Nacalai Tesque, Kyoto, Japan). BrdU was dissolved in $0.3 \times$ Danieau's solution $[19.3 \mathrm{mM} \mathrm{NaCl}, 0.23 \mathrm{mM} \mathrm{KCl}$, $0.13 \mathrm{mM} \mathrm{MgSO}_{4}, 0.2 \mathrm{mM} \mathrm{Ca}\left(\mathrm{NO}_{3}\right)_{2}, 1.7 \mathrm{mM}$ HEPES, pH 7.2]. 2-Phenoxyethanol was obtained from Wako Chemicals (Osaka, Japan).

\section{Comparative Transcriptome Analysis}

To identify genes that are differentially expressed in multiple rodent models of light-induced retinopathy, we analyzed three transcriptome datasets deposited in the Gene Expression
Omnibus (GEO; Barrett et al., 2009), designated GSE10528 (Rattner and Nathans, 2005), GSE22818 (Natoli et al., 2010), and GSE37773 (Hadziahmetovic et al., 2012). The raw data were normalized using the packages "affy" (Gautier et al., 2004) for GSE10528 and "oligo" (Carvalho et al., 2007) for GSE22818 and GSE37773 in Bioconductor (Gentleman et al., 2004). Probes with reliable signals were selected and subjected to RankProd (Breitling et al., 2004) analysis to identify differentially expressed genes (DEGs) in each model using a false discovery rate of $10 \%$ as the threshold. The gene symbols of the DEGs were converted to the human orthologs using Life Science Knowledge Bank (World Fusion, Tokyo, Japan), and UniProt IDs were added using the ID mapping tool of UniProt (UniProtConsortium, 2015). Venn diagrams showing the number of shared and unique DEGs in the three models were drawn using PINA4MS (Cowley et al., 2012) in Cytoscape (Shannon et al., 2003).

We identified functional networks significantly related to the 37 DEGs common to the three rodent models of light-induced retinopathy by using JEPETTO (Winterhalter et al., 2014) as the integrative analytical tool, with STRING (Jensen et al., 2009) for the protein interaction network analyses and Gene Ontology (Gene Ontology Consortium, 2015), InterPro (Mitchell et al., 2015), or BioCarta (Nishimura, 2001) for the functional analyses.

To identify TFs potentially regulating the 37 common DEGs, we used iRegulon (Janky et al., 2014), which has been used successfully to identify important TFs from gene lists (Nishimura et al., 2015b; Sasagawa et al., 2016). The predicted transcriptional regulators with normalized enrichment scores (NES) $>3$ are shown in Table S2.

\section{Zebrafish}

We used an albino line (Kelsh et al., 1996) obtained from the Max Planck Institute for Developmental Biology (Tübingen, Germany). Zebrafish were bred and maintained according to previously described methods (Westerfield, 2007; Nishimura et al., 2016). Briefly, zebrafish were raised at $28.5 \pm 0.5^{\circ} \mathrm{C}$ with a $14 \mathrm{~h} / 10 \mathrm{~h}$ light/dark cycle. Embryos were obtained and cultured in $0.3 \times$ Danieau's solution until 6 days post-fertilization (dpf). For modeling light-induced retinopathy, zebrafish were cultured in $0.3 \times$ Danieau's solution containing $200 \mu \mathrm{M}$ phenylthiourea.

\section{A Larval Zebrafish Model of Light-Induced Retinopathy}

Zebrafish at $3 \mathrm{dpf}$ were wrapped in aluminum foil and shielded from light for $48 \mathrm{~h}$. On day $5 \mathrm{dpf}$, the animals were transferred to 12 -well plates (7 larvae/3 ml medium/well) and incubated under normal conditions $\left(28^{\circ} \mathrm{C}, 14 \mathrm{~h}\right.$ [7 a.m. -10 p.m.] dim light $/ 10 \mathrm{~h}$ dark) or placed in a custom-made chamber (Hayashi Factory, Kyoto, Japan) to induce retinopathy (Figure S3). The chamber was $47 \times 47 \times 27 \mathrm{~cm}$ in width, length, and height, respectively, and was equipped with six fluorescent lamps (FL10ECW, Panasonic, Osaka, Japan) housed in the lid. Lamp specifications were $33 \mathrm{~cm}$ length, 520 lumen, 7200 kelvin, and emission peaks at 450,540 , and $610 \mathrm{~nm}$. The inner walls of the chamber were lined with mirrors to expose the zebrafish to light from all directions. The multi-well plates were placed on a water-cooled 
plate within the chamber to maintain the animals at $27^{\circ} \mathrm{C}$ during light exposure.

\section{TUNEL Staining}

Terminal deoxynucleotidyl transferase dUTP nick end labeling (TUNEL) was performed using an ApopTag Fluorescein In situ Apoptosis Detection Kit (Millipore, Billerica, MA, USA) according to the manufacturer's protocol. Briefly, zebrafish were exposed for $24 \mathrm{~h}$ to intense light (13,000 lux) or normal light conditions (14 h/10 h light/dark cycle) in medium with or without $2 \mu \mathrm{M}$ C646 and then fixed in 4\% paraformaldehyde in phosphate-buffered saline (PBS; Nacalai Tesque) at $4^{\circ} \mathrm{C}$ overnight. Animals were washed with PBS containing $0.1 \%$ Tween 20 (PBST), incubated in water containing $3 \% \mathrm{H}_{2} \mathrm{O}_{2}$ and $1 \% \mathrm{KOH}$ at room temperature for $30 \mathrm{~min}$, washed again with PBST, and incubated in $100 \%$ methanol at $-30^{\circ} \mathrm{C}$ overnight. Zebrafish were rehydrated, treated with proteinase $\mathrm{K}(40 \mu \mathrm{g} / \mathrm{ml})$ at $37^{\circ} \mathrm{C}$ for $1 \mathrm{~h}$, washed with $\mathrm{PBST}$, incubated in equilibration buffer at $37^{\circ} \mathrm{C}$ for $1 \mathrm{~h}$, and then incubated in working solution containing TdT enzyme and digoxigenin-labeled dNTP at $37^{\circ} \mathrm{C}$ for $1 \mathrm{~h}$. The animals were then washed and treated with fluorescein-labeled anti-digoxigenin IgG at $4^{\circ} \mathrm{C}$ overnight. Finally, zebrafish were washed once more with PBST and imaged with a SMZ25 stereomicroscope (Nikon, Tokyo, Japan) equipped with a GFP-BP filter. Quantitative analysis of the fluorescent images was performed using Volocity software (PerkinElmer, Waltham, MA, USA). The threshold fluorescence intensity for defining apoptotic areas of the retina was set at five standard deviations above the mean fluorescence intensity of the whole field of view.

\section{Whole-Mount Fluorescent Immunohistochemistry}

For the assessment of photoreceptor cells, zebrafish were exposed to intense or normal light conditions with or without $2 \mu \mathrm{M}$ C646 as described above, and then fixed in 4\% paraformaldehyde in PBS at $4^{\circ} \mathrm{C}$ overnight. Zebrafish were then washed with PBST, incubated in water containing $3 \% \mathrm{H}_{2} \mathrm{O}_{2}$ and $1 \% \mathrm{KOH}$ at room temperature for $30 \mathrm{~min}$, washed again with PBST, and incubated in $100 \%$ methanol at $-30^{\circ} \mathrm{C}$ overnight. After rehydration, zebrafish were treated with proteinase $\mathrm{K}(40 \mu \mathrm{g} / \mathrm{ml})$ at $28^{\circ} \mathrm{C}$ for $30 \mathrm{~min}$, washed with PBST, and then incubated in Blocking One Histo (Nacalai Tesque) at $4^{\circ} \mathrm{C}$ overnight. Animals were washed again with PBST and incubated in Can Get Signal Immunostain B solution (Toyobo, Osaka, Japan) containing antiZpr3 antibody (1:50, ZIRC, Eugene, OR, USA) at $4^{\circ} \mathrm{C}$ overnight. Zebrafish were washed with PBST and incubated in Can Get Signal Immunostain B solution (Toyobo) containing Alexa Fluor 488-conjugated anti-mouse IgG (1:500, Invitrogen, Carlsbad, CA) at $4^{\circ} \mathrm{C}$ overnight. After a final wash with PBST, animals were imaged with a SMZ25 stereomicroscope equipped with a GFP-BP filter. Quantitative analysis of the fluorescent images was performed using Volocity software. The threshold fluorescence intensity for identifying photoreceptor cell outer segments was set at four standard deviations above the mean fluorescence intensity of the whole field of view.

Retinal cell proliferation was measured as described above for the assessment of retinal cells, with the following modifications.
Zebrafish were exposed to intense or normal light conditions in $0.3 \times$ Danieau's solution containing $10 \mathrm{mM} \mathrm{BrdU}$ with or without $2 \mu \mathrm{M}$ C646, and then fixed, dehydrated, and rehydrated as described above. After rehydration, animals were treated with proteinase $\mathrm{K}(40 \mu \mathrm{g} / \mathrm{ml})$ at $37^{\circ} \mathrm{C}$ for $60 \mathrm{~min}$, washed with PBST, and then incubated in Blocking One Histo (Nacalai Tesque) at $4^{\circ} \mathrm{C}$ overnight. Animals were washed again with PBST and incubated in Can Get Signal Immunostain B solution (Toyobo) containing anti-BrdU antibody (1:100, Sigma) at $4^{\circ} \mathrm{C}$ overnight. Zebrafish were then processed as described above except the Alexa Fluor 488-conjugated anti-mouse IgG was used at 1:200 dilution. Finally, the animals were imaged with a SMZ25 stereomicroscope equipped with a GFP-BP filter. Quantitative analysis of the fluorescent images was performed using Volocity software. The threshold fluorescence intensity for BrdU was set at three standard deviations above the mean fluorescence intensity of the whole field of view.

\section{Statistical Analysis}

Statistical analysis was performed using Prism 6 (GraphPad, La Jolla, CA, USA). For the assessment of apoptosis (TUNEL staining) and photoreceptor cell outer segments, group means were compared by analysis of variance followed by Tukey's multiple comparisons test. For the assessment of BrdU-positive cells, group means were compared by the Kruskal-Wallis test followed by Dunn's multiple comparisons test. Data are shown as the mean \pm standard error (SEM).

\section{RESULTS}

\section{Identification of DEGs Common to the Three Rodent Models of Light-Induced Retinopathy}

To identify genes that are differentially expressed in multiple light-induced retinopathy models, we downloaded three transcriptome datasets from studies examining the retinas of rodents exposed to different light conditions. GSE10528 was from albino BALB/c mice $24 \mathrm{~h}$ after exposure to 6000 lux for $6 \mathrm{~h}$ (Rattner and Nathans, 2005), GSE22818 was from albino Sprague Dawley rats after exposure to 1000 lux for $24 \mathrm{~h}$ (Natoli et al., 2010), and GSE37773 was from albino BALB/c mice $4 \mathrm{~h}$ after exposure to 10,000 lux for $18 \mathrm{~h}$ (Hadziahmetovic et al., 2012). Using a false discovery rate of $10 \%$ as the threshold, we identified 261, 256, and 788 genes in GSE10528, GSE22818, and GSE37773, respectively, that were differentially expressed in animals exposed to intense light compared with normal light conditions (Table S1). Among the 39 DEGs that overlapped in the three light-induced retinopathy models (Figure 1), the change in expression was the same for 37 DEGs (Table 1), suggesting that they may be related to the pathophysiology of light-induced retinopathy.

\section{Identification of STAT1/3-IL6ST/OSMR As a Key Signaling Pathway in Light-Induced Retinopathy}

We next investigated which functional networks were significantly associated with the 37 common DEGs using 


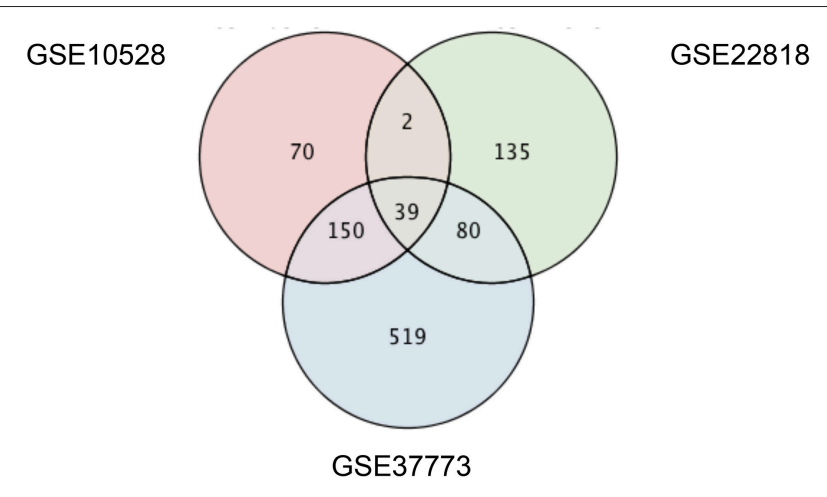

FIGURE 1 | Venn diagram of differentially expressed genes in the three rodent models of light-induced retinopathy. Transcriptome datasets from three different rodent models of light-induced retinopathy (GSE10528,

GSE22818, and GSE37773) were downloaded from a public database (GEO). Genes that were differentially expressed in the light-induced retinopathy vs. control groups were identified using a false discovery rate of $10 \%$ as the threshold. The number of differentially expressed genes in each transcriptome dataset and the overlap between datasets are shown.

the JEPETTO analytical plugin for Cytoscape (Winterhalter et al., 2014). JEPETTO performs integrated analyses using protein interaction networks such as STRING (Jensen et al., 2009) and various databases such as Gene Ontology (Gene Ontology Consortium, 2015), InterPro (Mitchell et al., 2015), and BioCarta (Nishimura, 2001) to find functional networks significantly related to a given gene list. Using JEPETTO with InterPro, we found that "STAT transcription factor, coiled coil" was significantly associated with the 37 common DEGs (XD score 0.56 and $q$-value $1.7 \times 10^{-1}$, Figure 2 ). Among the 12 genes included in the "STAT transcription factor, coiled coil" network, 7 genes (STAT1, STAT3, IL6ST, OSMR, JUN, FOS, and IRF9) were significantly upregulated in the three rodent models of light-induced retinopathy. STAT1 and STAT3 can form homodimers or heterodimers (reviewed in Kisseleva et al., 2002). A coiled coil domain important for protein-protein interactions is conserved in all members of the STAT family (reviewed in Reich, 2013). Interleukin 6 signal transducer (IL6ST), also known as GP130, can form heterodimers with the oncostatin M receptor (OSMR; Hermanns et al., 1999). Activation of STAT3 increases transcription of IL6ST (O'Brien and Manolagas, 1997) and OSMR (Traber et al., 2015) and, in turn, activation of IL6ST/OSMR leads to STAT1/3 activation (Dreuw et al., 2004). These findings suggest that STAT1/3 and IL6ST/OSMR may form a positive feedback loop. Using JEPETTO in combination with Gene Ontology and BioCarta, we found that "IL 6 signaling pathway" (XD score 0.70 and $q$-value $5.8 \times 10^{-4}$, Figure S1) and "growth factor binding" (XD score 0.50 and $q$-value $2.6 \times 10^{-2}$, Figure S2), respectively, were significantly associated with the 37 common DEGs. STAT1, STAT3, IL6ST, OSMR, JUN, FOS, and IRF9 are represented in the "STAT transcription factor, coiled coil," "IL 6 signaling pathway," and "growth factor binding" networks. These results suggest that STAT1/3-IL6ST/OSMR may be a key signaling pathway in the pathophysiology of light-induced retinopathy.

\section{Identification of EP300 As a Key Transcription Factor in Light-Induced Retinopathy}

We hypothesized that the 37 DEGs common to the three rodent models of light-induced retinopathy might be regulated by specific TFs. To test this, we analyzed the gene sets using iRegulon, which exploits the fact that genes co-regulated by the same TF contain common TF binding sites and uses gene sets derived from ENCODE ChIP-seq data (Gerstein et al., 2012; Janky et al., 2014). Using iRegulon, we identified six TFs with significant NES ( $>3$ ), suggesting that they regulate the expression of common DEGs (Table S2). EP300, STAT1, and STAT3 were the first, second, and third ranked TFs by significance. iRegulon identified 28 genes (Figure 3A), 6 genes (Figure 3B), and 20 genes (Figure 3C) as potential transcriptional targets of EP300, STAT1, and STAT3, respectively. The union of the EP300, STAT1, and STAT3 networks and their potential transcriptional targets is shown in Figure 3D. Of the potential targets, 17 genes, including STAT1, STAT3, IL6ST, and OSMR, were targets of EP300 and either STAT1 or STAT3. These results suggest that EP300 and STAT1/3 may regulate the expression of the 16 genes cooperatively and that EP300 may be a key TF in light-induced retinopathy.

\section{Inhibition of EP300 Increases Apoptosis in the Zebrafish Model of Light-Induced Retinopathy}

To examine the function of EP300 in light-induced retinopathy, we developed a novel model using larval zebrafish (Figures S3 and Figure 4A). Larval zebrafish were first shielded from light for $48 \mathrm{~h}$ between 3 and $5 \mathrm{dpf}$ and then exposed to intense light $(13,000$ lux for $24 \mathrm{~h})$ or housed under normal conditions $(14 \mathrm{~h}$ 250 lux/10 h dark) in the absence or presence of C646, a specific inhibitor of EP300 (Bowers et al., 2010). At the end of the $24 \mathrm{~h}$ incubation, retinal apoptosis was examined by TUNEL staining. The retinas of zebrafish exposed to intense light had significantly higher apoptosis levels than the retinas of zebrafish housed under normal conditions (Figures 4B,C). Moreover, C646 treatment further increased the level of apoptosis in the retinas of zebrafish exposed to intense light, whereas it had no effect on zebrafish housed under normal light conditions (Figure 4C). To exclude the possibility that C646 might induce non-specific cell death following light exposure, we measured apoptosis in the forebrain. However, we found no significant difference in the apoptotic signals between the forebrains of zebrafish exposed to intense light with and without C646 treatment (data not shown). These results suggest that activated EP300 may protect the retina by reducing apoptosis during intense light exposure.

\section{Inhibition of EP300 Increases Loss of Photoreceptor Cell Outer Segments in the Zebrafish Model of Light-Induced Retinopathy}

To further investigate the role of EP300 in retinal protection, we performed whole-mount immunohistochemical staining of 
TABLE 1 | DEGs common to the three rodent models of light-induced retinopathy.

\begin{tabular}{|c|c|c|c|c|c|c|c|}
\hline \multirow[t]{2}{*}{ Symbol } & \multirow[t]{2}{*}{ Name } & \multicolumn{2}{|c|}{ GSE10528 } & \multicolumn{2}{|c|}{ GSE22818 } & \multicolumn{2}{|c|}{ GSE37773 } \\
\hline & & log(light/cnt) & FDR & log(light/cnt) & FDR & $\log ($ light/cnt) & FDR \\
\hline A2M & Alpha-2-macroglobulin & 2.39 & 0.00 & 0.83 & 0.06 & 2.70 & 0.00 \\
\hline ADAMTS1 & ADAM metallopeptidase with thrombospondin type 1 motif 1 & 1.48 & 0.01 & 1.40 & 0.01 & 1.35 & 0.00 \\
\hline AHR & Aryl hydrocarbon receptor & 1.01 & 0.07 & 1.84 & 0.00 & 1.32 & 0.00 \\
\hline AQP1 & Aquaporin 1 & -1.13 & 0.01 & -1.32 & 0.00 & -0.74 & 0.02 \\
\hline CD44 & CD44 molecule & 2.34 & 0.00 & 1.26 & 0.01 & 1.73 & 0.00 \\
\hline $\mathrm{CP}$ & Ceruloplasmin & 2.14 & 0.00 & 1.25 & 0.02 & 1.95 & 0.00 \\
\hline DUSP6 & Dual specificity phosphatase 6 & 1.69 & 0.00 & 0.96 & 0.04 & 0.73 & 0.02 \\
\hline EBPL & Emopamil binding protein-like & -0.92 & 0.04 & -1.18 & 0.00 & -0.96 & 0.00 \\
\hline EDN2 & Endothelin 2 & 3.69 & 0.00 & 0.99 & 0.03 & 0.76 & 0.02 \\
\hline FOS & FBJ murine osteosarcoma viral oncogene homolog & 1.14 & 0.08 & 1.28 & 0.01 & 1.00 & 0.00 \\
\hline IFITM2 & Interferon induced transmembrane protein 2 & 1.31 & 0.01 & 1.64 & 0.01 & 2.45 & 0.00 \\
\hline IL6ST & Interleukin 6 signal transducer & 1.15 & 0.03 & 1.10 & 0.02 & 1.21 & 0.00 \\
\hline IPO5 & Importin 5 & 1.25 & 0.01 & 0.98 & 0.03 & 1.13 & 0.00 \\
\hline IRF9 & Interferon regulatory factor 9 & 1.86 & 0.00 & 2.35 & 0.00 & 2.30 & 0.00 \\
\hline ITGB1 & Integrin, beta 1 & 1.00 & 0.07 & 0.76 & 0.10 & 0.59 & 0.05 \\
\hline JUN & Jun proto-oncogene & 1.57 & 0.00 & 2.04 & 0.00 & 1.42 & 0.00 \\
\hline LGALS3 & Lectin, galactoside-binding, soluble, 3 & 3.14 & 0.00 & 1.30 & 0.02 & 0.80 & 0.01 \\
\hline LITAF & Lipopolysaccharide-induced TNF factor & 0.97 & 0.08 & 1.91 & 0.00 & 0.68 & 0.03 \\
\hline MSN & Moesin & 2.19 & 0.00 & 1.03 & 0.04 & 1.97 & 0.00 \\
\hline$N R L$ & Neural retina leucine zipper & -1.04 & 0.02 & -1.12 & 0.01 & -0.81 & 0.01 \\
\hline OPTN & Optineurin & -0.91 & 0.04 & -0.96 & 0.02 & -0.69 & 0.03 \\
\hline OSMR & Oncostatin M receptor & 3.04 & 0.00 & 2.59 & 0.00 & 2.61 & 0.00 \\
\hline PDPN & Podoplanin & 2.78 & 0.00 & 1.99 & 0.00 & 1.79 & 0.00 \\
\hline PITPNM3 & PITPNM family member 3 & -1.37 & 0.00 & -0.84 & 0.08 & -0.71 & 0.02 \\
\hline PROS1 & Protein S (alpha) & 0.96 & 0.09 & 1.06 & 0.02 & 1.72 & 0.00 \\
\hline $\mathrm{RDH} 10$ & retinol dehydrogenase 10 (all-trans) & 1.00 & 0.07 & 1.58 & 0.00 & 0.50 & 0.10 \\
\hline REEP6 & Receptor accessory protein 6 & -1.23 & 0.01 & -1.70 & 0.00 & -1.30 & 0.00 \\
\hline S100A10 & S100 calcium binding protein A10 & 2.44 & 0.00 & 0.91 & 0.06 & 2.03 & 0.00 \\
\hline S100A6 & S100 calcium binding protein A6 & 2.45 & 0.00 & 1.16 & 0.03 & 3.62 & 0.00 \\
\hline SLC14A1 & Solute carrier family 14, member 1 & 2.17 & 0.00 & 1.41 & 0.01 & 2.57 & 0.00 \\
\hline SLC24A1 & Solute carrier family 24 member 1 & -0.85 & 0.06 & -0.98 & 0.03 & -0.65 & 0.04 \\
\hline STAT1 & Signal transducer and activator of transcription 1, 91kDa & 2.00 & 0.00 & 1.60 & 0.01 & 2.17 & 0.00 \\
\hline STAT3 & Signal transducer and activator of transcription 3 & 1.92 & 0.00 & 2.03 & 0.00 & 1.96 & 0.00 \\
\hline SUSD3 & Sushi domain containing 3 & -1.06 & 0.02 & -1.18 & 0.00 & -0.74 & 0.02 \\
\hline TAGLN2 & Transgelin 2 & 0.98 & 0.09 & 0.96 & 0.05 & 1.03 & 0.00 \\
\hline TARS & Threonyl-tRNA synthetase & 1.06 & 0.05 & 1.22 & 0.01 & 0.66 & 0.03 \\
\hline TRIM25 & Tripartite motif containing 25 & 1.01 & 0.07 & 0.97 & 0.04 & 1.53 & 0.00 \\
\hline
\end{tabular}

The changes of gene expression increased and decreased in light-induced retinopathy are shown in red and blue, respectively.

larval zebrafish with light-induced retinopathy (Figure 5A) using anti-Zpr3 antibody, which detects both rod and cone photoreceptor cell outer segments (Yin et al., 2012). As shown in Figure 5B, the fluorescent signal from the photoreceptor cell outer segments, especially in the area between the rim and the lens, was decreased in zebrafish exposed to intense light in the presence of C646. Quantitative analysis of the fluorescent signal revealed a significant reduction in the area of photoreceptor cell outer segments in zebrafish exposed to intense light in the presence of C646 compared with zebrafish housed under normal conditions (Figure 5C). The retina diameters were not significantly different among the four groups (data not shown). These results support a role for EP300 in protecting the retina from light-induced damage, at least in zebrafish.

\section{Inhibition of EP300 Increases BrdU Incorporation in Putative Müller Cells in the Zebrafish Model of Light-Induced Retinopathy}

Following retinal injury, zebrafish Müller glia proliferate and differentiate into the retinal cell types lost through injury 


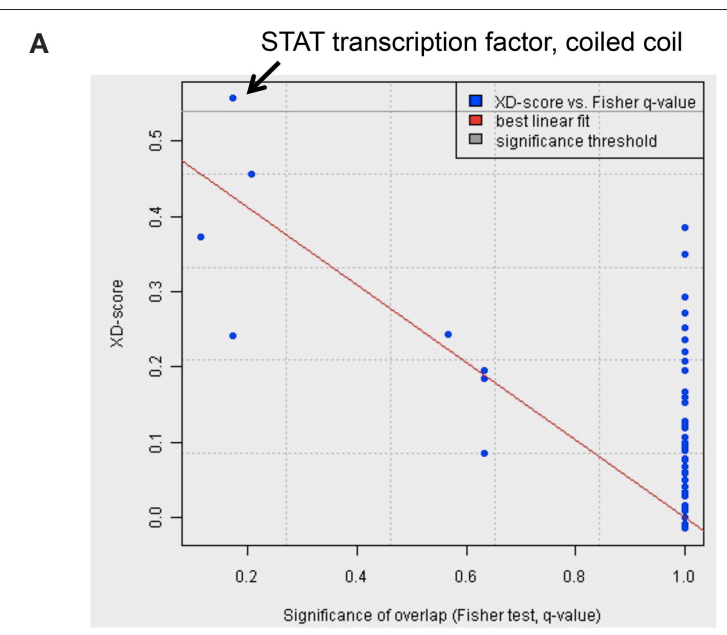

B

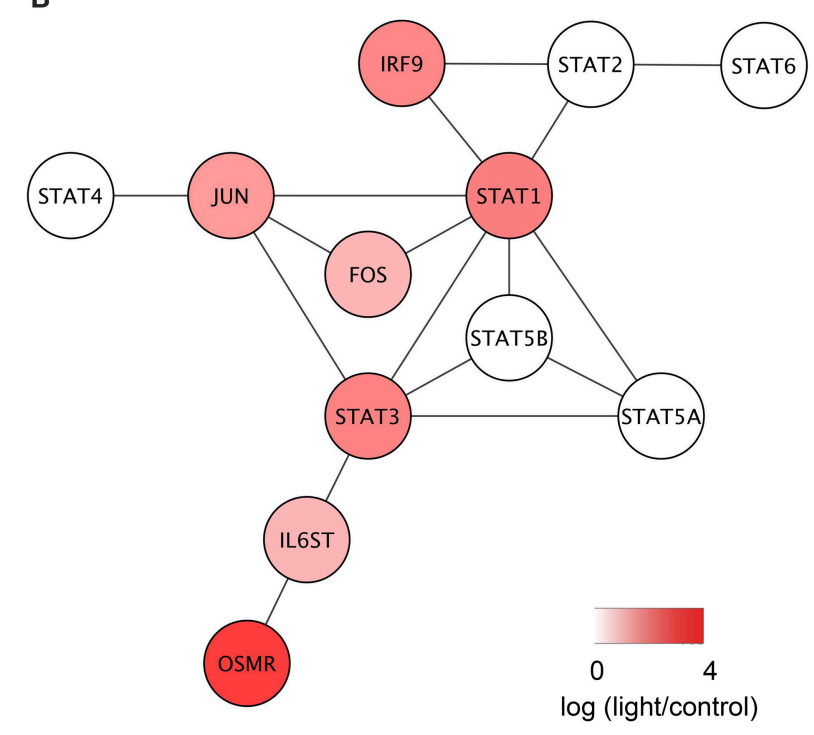

FIGURE 2 | Identification of "STAT transcription factor, coiled coil" as the domain most significantly associated with the DEGs common to the three rodent models of light-induced retinopathy. (A) Scatter plot of domains in InterPro based on the network-based association score (XD score) and the significance of overlap ( $q$-value) using the 37 common DEGs as the input in JEPETTO. The most significant domain was "STAT transcription factor, coiled coil." (B) The "STAT transcription factor, coiled coil" network identified by JEPETTO. The seven genes with increased expression in the light-induced retinopathy models are shown in red.

(Lenkowski and Raymond, 2014). To determine whether Müller cells proliferate in zebrafish with light-induced retinopathy, zebrafish were immersed in medium containing BrdU with or without $\mathrm{C} 646$ and/or exposed to intense light for 1 day (Figure 6A), after which, whole-mount immunohistochemical staining with anti-BrdU antibody was performed. As shown in Figures $6 \mathrm{~B}, \mathrm{C}$, there was a significant increase in the appearance of BrdU-positive cells with an elongated morphology, consistent with the morphology of proliferating Müller cells (Yurco and Cameron, 2005), in zebrafish treated with C646 and exposed to intense light. These results suggest that inhibition of EP300 may increase retinal injury in the zebrafish model of light-induced retinopathy, thereby stimulating the proliferation of Müller glia.

\section{DISCUSSION}

The results of this study suggest that (i) the STAT1/3IL6ST/OSMR axis is a key signaling pathway in three rodent models of light-induced retinopathy, (ii) EP300 is a key upstream regulator of the STAT1/3-IL6ST/OSMR axis, and (iii) EP300 plays a protective role in a larval zebrafish model of light-induced retinopathy.

\section{The STAT1/3-IL6ST/OSMR Axis Is a Key Signaling Pathway in Light-Induced Retinopathy}

We identified 37 genes differentially regulated in the three rodent models of light-induced retinopathy, and found significant enrichment of these genes in "STAT transcription factor, coiled coil," "IL 6 signaling pathway," and "growth factor binding" networks. STAT1, STAT3, IL6ST, and OSMR were represented in all three networks and the expression of these genes was increased in the three rodent models studied here.

Previous work has demonstrated that the expression of STAT1 and STAT3 is increased in light-induced retinopathy (Samardzija et al., 2006) and that the increase in STAT3 is not observed in leukemia inhibitory factor (LIF) null mice (Bürgi et al., 2009). In the retina of BALB/c mice, expression of LIF, which is a member of the IL-6 family (Heinrich et al., 2003), is markedly increased by light exposure, peaking at 6 to $12 \mathrm{~h}$ after exposure (Samardzija et al., 2006). These findings suggest that the increased expression of STAT1 and STAT3 observed in the three light-induced retinopathy models examined here may be mediated by LIF. STAT1 and STAT3 can bind to the promoter of IL6ST as homodimers or heterodimers and positively regulate its transcription (O'Brien and Manolagas, 1997). Activation of STAT3 also increases transcription of OSMR (Traber et al., 2015). Therefore, the retinopathy-associated increase in IL6ST and OSMR detected here may be a result of STAT1/3-mediated transcriptional activation.

IL6ST and OSMR have also been shown to form heterodimers (Hermanns et al., 1999), and activation of such heterodimers leads to STAT1/3 activation (Dreuw et al., 2004). Given that STAT1, STAT3, IL6ST, and OSMR are all differentially upregulated in the models studied here, these reports are consistent with the hypothesis that the STAT1/3-IL6ST/OSMR axis is activated in light-induced retinopathy. Knockout of IL6ST increases photoreceptor cell death not only in a light-induced retinal damage model (Ueki et al., 2009) but also in other photoreceptor degeneration models such as retinitis pigmentosa and age-related macular degeneration (Rhee et al., 2013). Photoreceptor-specific deletion of STAT3 accelerates photoreceptor degeneration, whereas the expression of dominant active STAT3 improves photoreceptor survival in two models of retinitis pigmentosa (Jiang et al., 2014). Collectively, these findings are consistent with the possibility that STAT1/3-IL6ST/OSMR axis may be activated in light-induced 


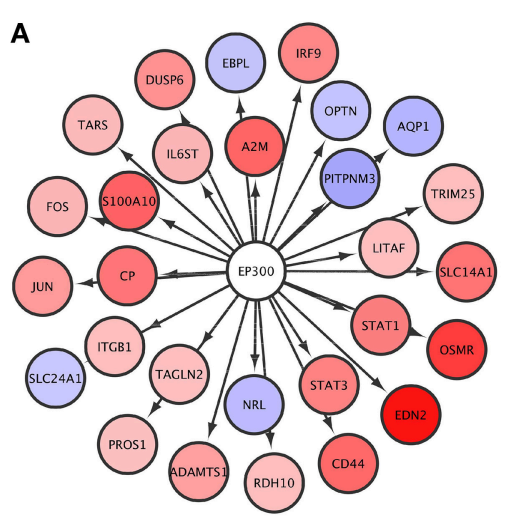

B

C
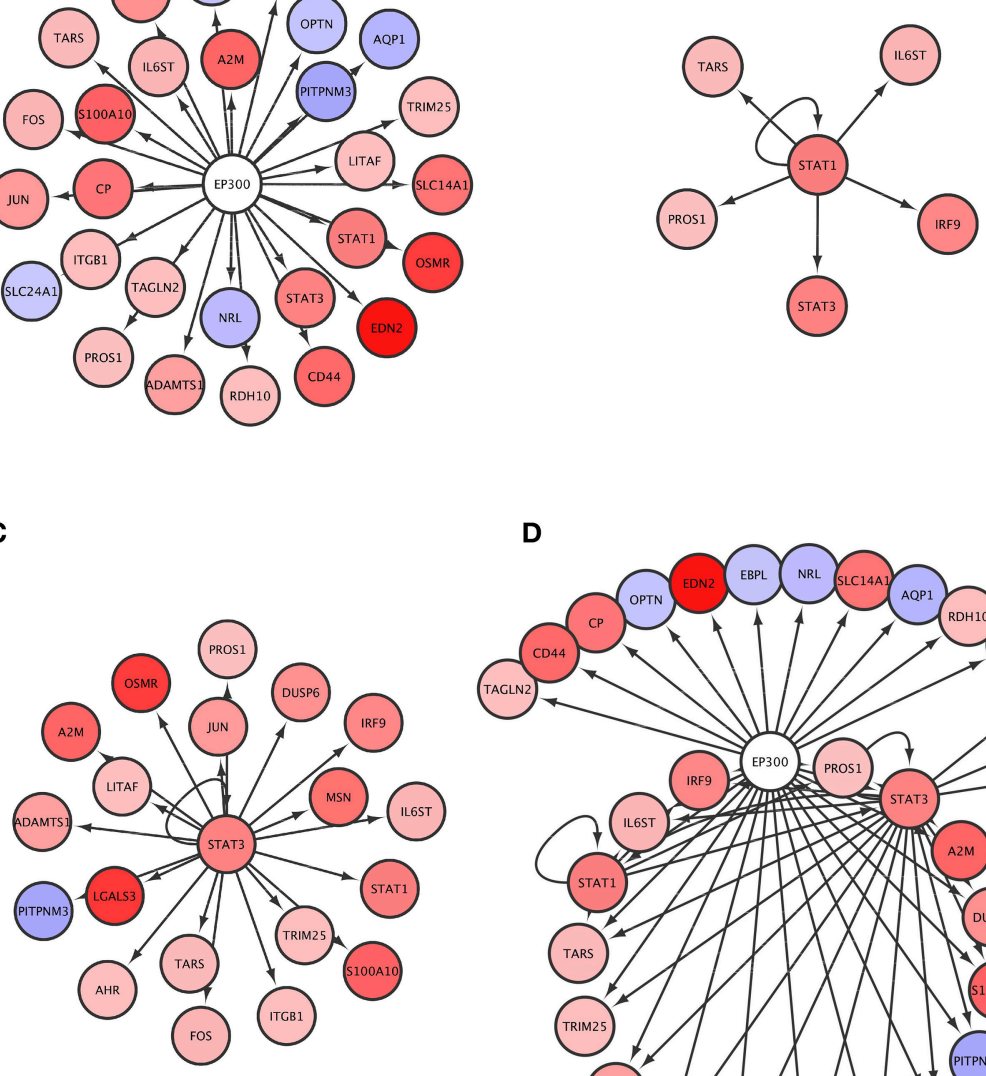

D
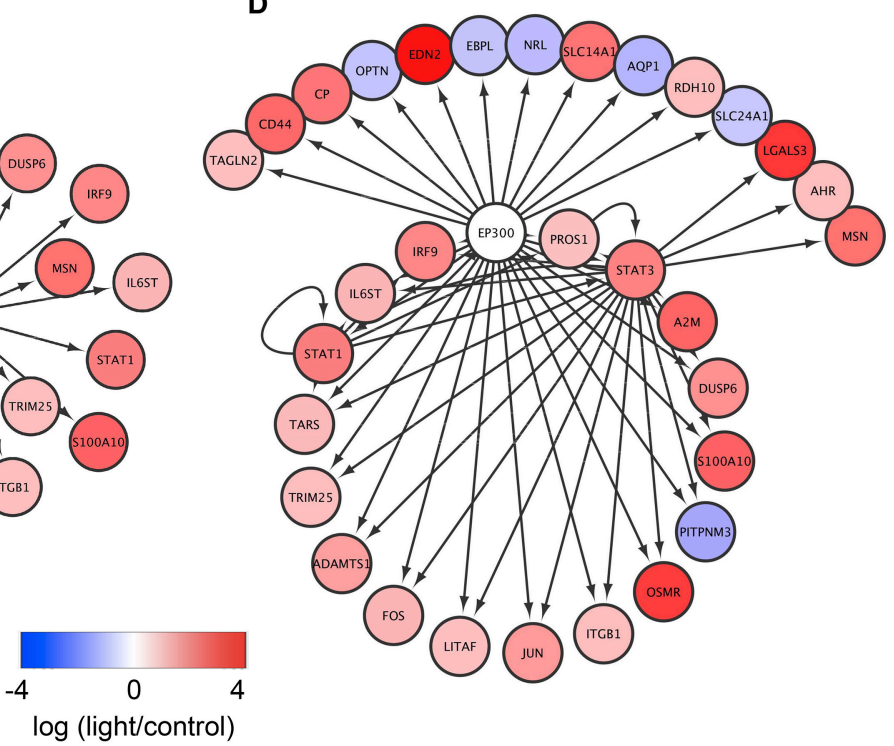

FIGURE 3 | Identification of EP300, STAT1, and STAT3 as the key transcription factors potentially regulating the DEGs common to the three rodent models of light-induced retinopathy. (A-C) The DEGs common to the three models and potentially regulated by EP300 (A), STAT1 (B), and STAT3 (C). (D) The union of the EP300, STAT1, and STAT3 networks and their potential targets.

retinopathy as an endogenous anti-apoptotic signaling pathway.

\section{EP300 Is a Key Upstream Regulator of the STAT1/3-IL6ST/OSMR AXis}

Our study identified 16 genes, including STAT1, STAT3, IL6ST, and OSMR, as potential transcriptional targets of EP300 and either STAT1 or STAT3, suggesting that EP300 and STAT1/3 may act cooperatively to regulate expression of the 16 genes in light-induced retinopathy.

EP300 is a transcriptional cofactor with intrinsic acetyltransferase activity (reviewed in Ghosh and Varga, 2007). The activity of STAT1 and STAT3 is known to be regulated by EP300-dependent acetylation (Yuan et al., 2005; Hou et al., 2008; Kotla and Rao, 2015; reviewed in Zhuang, 2013), and complexes of EP300 and STAT3 can bind to the promoters of IL6ST (Chen et al., 2008) and glial fibrillary acidic protein
(GFAP) (Nakashima et al., 1999) and regulate their expression. In addition, inhibition of EP300 by C646 reduces phosphorylation of STAT3 at tyrosine 705 by angiotensin II (Ni et al., 2014). Tyrosine 705 phosphorylation induces STAT3 translocation to the nucleus and activates its transcriptional activity (Wen et al., 1995). Taken together with these reports, our findings support a role for EP300 as a key modulator of the anti-apoptotic effects of the endogenously activated STAT1/3-IL6ST/OSMR axis in light-induced retinopathy.

Acetylation of STAT3 can be regulated not only by HATs such as EP300 and CREB binding protein but also by HDACs (reviewed in Choi and Reddy, 2011; Zhuang, 2013). Sirtuin 1, a member of the sirtuin family of HDACs, deacetylates STAT3, resulting in decreased STAT3 tyrosine phosphorylation and transcriptional activation (Nie et al., 2009). Excessive HDAC activation plays a critical role in neurodegeneration in a mouse model of retinitis pigmentosa (Sancho-Pelluz et al., 
A

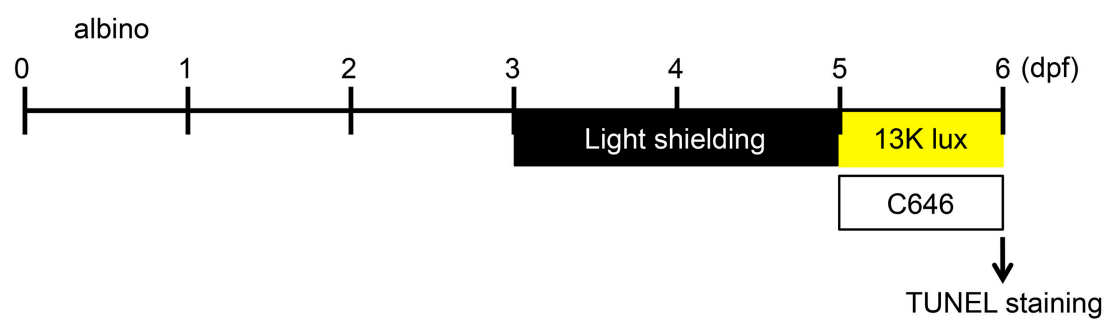

B
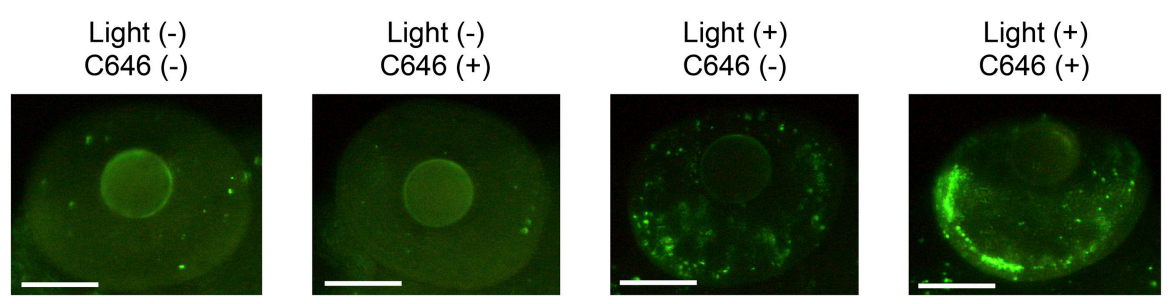

C

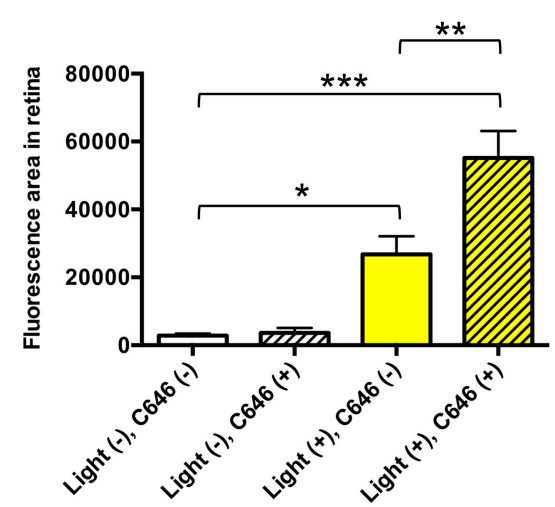

FIGURE 4 | Inhibition of EP300 increases retinal apoptosis in a larval zebrafish model of light-induced retinopathy. (A) Protocol for light-induced retinal damage in larval zebrafish. Zebrafish are shielded from light between 3 and 5 days post-fertilization (dpf) and then exposed to normal conditions (14 $\mathrm{h} 250$ lux/10 $\mathrm{h}$ dark) or intense light (13,000 lux) in the presence or absence of $2 \mu \mathrm{M} \mathrm{C646}$ for $24 \mathrm{~h}$ at $27^{\circ} \mathrm{C}$. After light exposure, whole-mount TUNEL staining was performed. (B) Representative images of TUNEL staining in the retina of zebrafish exposed to intense light (indicated as light+) or normal light conditions (light-). Scale bars, $100 \mu \mathrm{m}$. (C) Quantitative analysis of retinal apoptosis in zebrafish exposed to the conditions shown in (B). ${ }^{\star} p<0.01,{ }^{* *} p<0.001,{ }^{* \star *} p<0.0001$. Data are the mean \pm SEM of 13-14 zebrafish/group.

2010), and several HDAC inhibitors have neuroprotective effects in retinal degenerative diseases (reviewed in Zhang et al., 2015). Thus, activation of HATs and/or inhibition of HDACs to increase STAT3 acetylation may be a potential therapeutic approach for retinal degenerative diseases resembling lightinduced retinopathy.

\section{EP300 Is Protective in a Larval Zebrafish Model of Light-Induced Retinopathy}

In this study, we demonstrated that inhibition of EP300 by C646 significantly increased (i) retinal apoptosis, (ii) photoreceptor damage, and (iii) proliferation of putative Müller cells in a larval zebrafish model of light-induced retinopathy.

Inhibition of photoreceptor apoptosis is an important strategy to protect the morphology and function of the retina in retinal degenerative diseases (Samardzija et al., 2006). Application of ciliary neurotrophic factor (CNTF) and fibroblast growth factor 2 (FGF2) to the retina significantly reduces apoptosis (Kassen et al., 2009; Kolomeyer and Zarbin, 2014) and increases the survival of photoreceptor cells in lightinduced retinopathy (reviewed in Organisciak and Vaughan, 2010). Moreover, the interaction between EP300 and STAT3 is increased by treatment with CNTF (Hong and Song, 2014) or FGF2 (Cheng et al., 2005), suggesting a possible mechanism whereby CNTF and FGF2 reduce apoptosis and protect photoreceptor cells from light-induced retinopathy. Further studies are required to find small molecules that can activate EP300 and to examine their effects on apoptosis in light-induced retinopathy.

In this study, we described a new larval zebrafish model of light-induced retinopathy. Although similar models have 
A

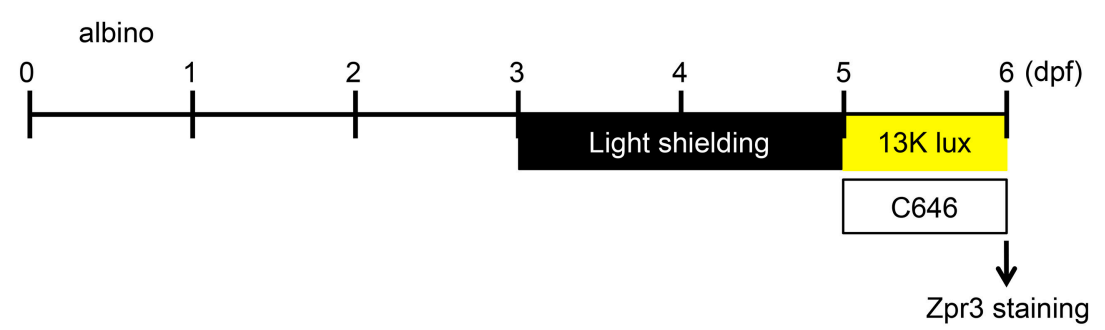

B

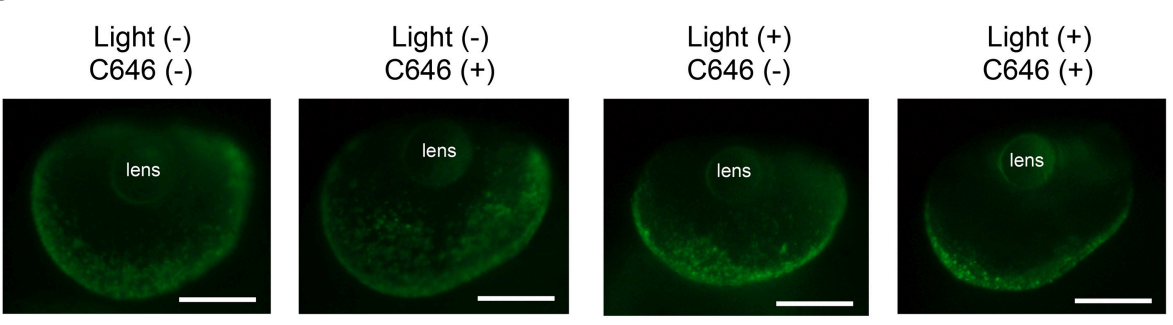

C

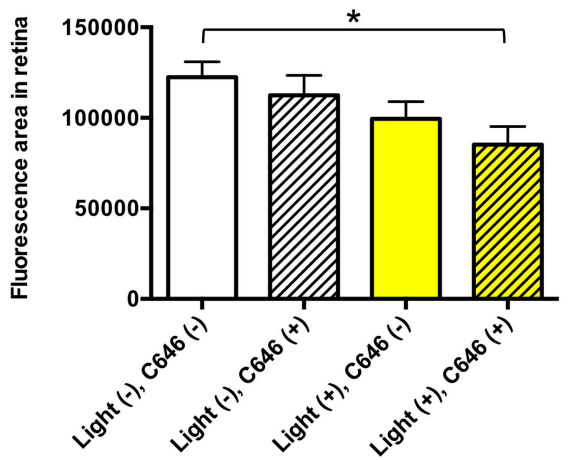

FIGURE 5 | Inhibition of EP300 reduces the photoreceptor cell outer segments in a zebrafish model of light-induced retinopathy. (A) Protocol for light-induced retinal damage in larval zebrafish, as described for Figure 4A. After light exposure, whole-mount immunohistochemical staining with anti-Zpr3 antibody was performed. (B) Representative images of anti-Zpr3 antibody staining of zebrafish exposed to normal or intense light. Scale bars, $100 \mu \mathrm{m}$. (C) Quantitative analysis of photoreceptor cell outer segments of zebrafish exposed to the conditions shown in (B). ${ }^{*} p<0.05$. Data are the mean \pm SEM of $n=24$ zebrafish/group for light-/C646- and light-/C646+, $n=17$ for light+/C646-, and $n=22$ for light+/C646+.

been successfully implemented with adult zebrafish (reviewed in Gorsuch and Hyde, 2014), we are aware of only one other report demonstrating that larval zebrafish can be used to model light-induced retinopathy (Meyers et al., 2012). In that study, larval zebrafish in a $50 \mathrm{ml}$ beaker were exposed to light using a liquid fiber optic light line connected to a metalhalide microscope illuminator. In our system, four multi-well plates can be illuminated at the same time, allowing chemical screening to be performed in a relatively high-throughput manner.

The zebrafish and human retinas are very similar (reviewed in Chhetri et al., 2014; Nishimura et al., 2015a). The neuronal cell bodies in both zebrafish and humans are precisely organized in three major laminae that are separated by plexiform layers. Similar to humans, zebrafish are diurnal. Zebrafish also have blood-brain and blood-retinal barriers (Watanabe et al., 2012; Nishimura et al., 2013). There are a few differences, however, between the visual systems of zebrafish and humans; for example, zebrafish have lateral eyes with reduced binocular overlap in the visual fields, less densely populated ganglion cells, absent fovea, and tetrachromatic vision (Chhetri et al., 2014). Understanding the similarities and differences between zebrafish and human vision is critical if the zebrafish model is to be useful for understanding the pathophysiology of human retinal diseases and for developing novel therapies. Many transgenic lines expressing fluorescent proteins in specific retinal subpopulations have also been developed and are available through public resources, enabling various approaches to in vivo imaging of the retina of larval zebrafish (Nishimura et al., 2015a). Knockout and knockin of specific genes can also be performed using transcription activator-like effector nuclease (TALEN) and clustered regularly interspaced short palindromic repeats (CRISPR)-Cas9 systems (Auer and Del Bene, 2014). When used in combination 
A

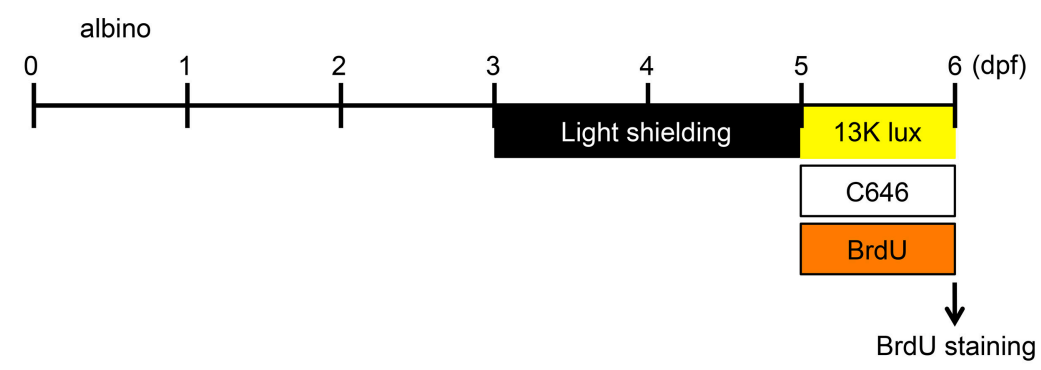

B

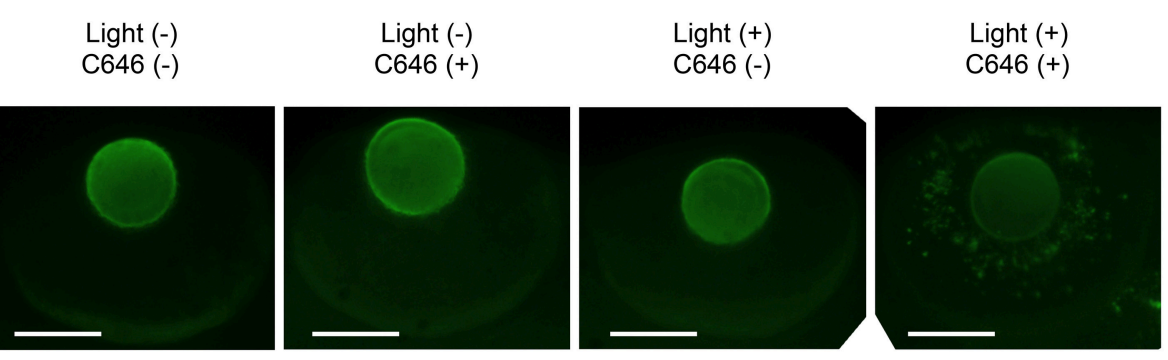

C

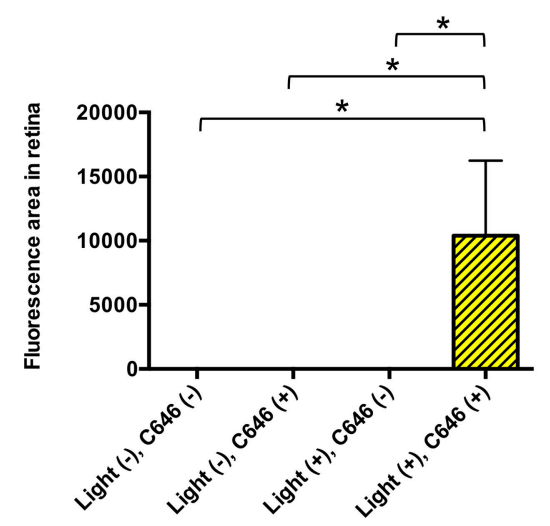

FIGURE 6 | Inhibition of EP300 increases BrdU incorporation in putative Müller cells in the zebrafish model of light-induced retinopathy. (A) Protocol for light-induced retinal damage in larval zebrafish, as described for Figure 4A. After light exposure, whole-mount immunohistochemical staining with anti-BrdU antibody was performed. (B) Representative images of anti-BrdU antibody staining of zebrafish exposed to normal or intense light. Scale bars, $100 \mu \mathrm{m}$. (C) Quantitative analysis of BrdU-positive cells in the retinas of zebrafish exposed to the conditions shown in (B). ${ }^{*} p<0.05$. Data are the mean \pm SEM of $n=8$ for all groups.

with these advanced technologies, the larval zebrafish model of light-induced retinopathy developed in this study will be a powerful tool in the search for therapeutic compounds that can protect photoreceptors from degenerative retinal diseases.

\section{AUTHOR CONTRIBUTIONS}

YN conceived the study, performed the bioinformatics analyses, developed the larval zebrafish model of light-induced retinopathy, and wrote the manuscript. RK developed the larval zebrafish model of light-induced retinopathy and validated the effect of EP300. YA, SS, SM, MY, SO, and KK helped with the experiments. HY, KM, SY, KT, MS, and HH provided essential conceptual help in fabricating the light-induced retinopathy chamber. TT conceived the study, developed the larval zebrafish model of light-induced retinopathy, and wrote the manuscript.

\section{FUNDING}

This work was supported in part by JSPS KAKENHI (25670127, 15K15051, 24510069), JST A-step (AS262Z00004Q), and the Long-range Research Initiative of the Japan Chemical Industrial Association (13_PT01-01).

\section{ACKNOWLEDGMENTS}

We thank Junko Koiwa, Hiroko Nakayama, Yuka Hayakawa, Yuka Takahashi, Chizuru Hirota, and Michiko Ariyoshi for assistance with the experiments, and Rie Ikeyama and Yuka Mizutani for secretarial support. 


\section{SUPPLEMENTARY MATERIAL}

The Supplementary Material for this article can be found online at: http://journal.frontiersin.org/article/10.3389/fphar. 2016.00126

Table S1 | DEGs in each rodent model of light-induced retinopathy. S1-1 DEGs in a mouse model of light-induced retinopathy (GSE10528). S1-2 DEGs in a rat model of light-induced retinopathy (GSE22818). S1-3 DEGs in a mouse model of light-induced retinopathy (GSE37773).

Table S2 | Transcription factors potentially regulating DEGs common to the three rodent models of light-induced retinopathy.

Figure S1 | Identification of "IL 6 signaling pathway" as the pathway most significantly associated with the DEGs common to the three rodent models of light-induced retinopathy. (A) Scatter plot of pathways in Biocarta based on the network-based association score (XD score) and the significance of overlap ( $q$-value) using the 37 common DEGs as the input in JEPETTO. The most significant domain was "IL 6 signaling pathway." (B) The "IL 6 signaling pathway" network identified by JEPETTO. The nine genes with increased expression in the light-induced retinopathy models are shown in red.

Figure S2 | Identification of "growth factor binding" as the molecular function most significantly associated with the DEGs common to the three rodent models of light-induced retinopathy. (A) Scatter plot of molecular functions in Gene Ontology based on the network-based association score (XD score) and the significance of overlap ( $q$-value) using the 37 common DEGs as the input in JEPETTO. The most significant domain was "growth factor binding." (B) The "growth factor binding" network identified by JEPETTO. The eight genes with increased expression in the light-induced retinopathy models are shown in red.

Figure S3 | The chamber fabricated for the larval zebrafish model of light-induced retinopathy.

\section{REFERENCES}

Auer, T. O., and Del Bene, F. (2014). CRISPR/Cas9 and TALENmediated knock-in approaches in zebrafish. Methods 69, 142-150. doi: 10.1016/j.ymeth.2014.03.027

Barrett, T., Troup, D. B., Wilhite, S. E., Ledoux, P., Rudnev, D., Evangelista, C., et al. (2009). NCBI GEO: archive for high-throughput functional genomic data. Nucleic Acids Res. 37, D885-D890. doi: 10.1093/nar/gkn764

Bowers, E. M., Yan, G., Mukherjee, C., Orry, A., Wang, L., Holbert, M. A., et al. (2010). Virtual ligand screening of the p300/CBP histone acetyltransferase: identification of a selective small molecule inhibitor. Chem. Biol. 17, 471-482. doi: 10.1016/j.chembiol.2010.03.006

Breitling, R., Armengaud, P., Amtmann, A., and Herzyk, P. (2004). Rank products: a simple, yet powerful, new method to detect differentially regulated genes in replicated microarray experiments. FEBS Lett. 573, 83-92. doi: 10.1016/j.febslet.2004.07.055

Bürgi, S., Samardzija, M., and Grimm, C. (2009). Endogenous leukemia inhibitory factor protects photoreceptor cells against light-induced degeneration. Mol. Vis. $15,1631-1637$

Carvalho, B., Bengtsson, H., Speed, T. P., and Irizarry, R. A. (2007). Exploration, normalization, and genotype calls of high-density oligonucleotide SNP array data. Biostatistics 8, 485-499. doi: 10.1093/biostatistics/kxl042

Chen, X., Xu, H., Yuan, P., Fang, F., Huss, M., Vega, V. B., et al. (2008). Integration of external signaling pathways with the core transcriptional network in embryonic stem cells. Cell 133, 1106-1117. doi: 10.1016/j.cell.2008.04.043

Chen, Y., Perusek, L., and Maeda, A. (2016). Autophagy in light-induced retinal damage. Exp. Eye Res. 144, 64-72. doi: 10.1016/j.exer.2015.08.021

Cheng, L. C., Tavazoie, M., and Doetsch, F. (2005). Stem cells: from epigenetics to microRNAs. Neuron 46, 363-367. doi: 10.1016/j.neuron.2005.04.027

Chhetri, J., Jacobson, G., and Gueven, N. (2014). Zebrafish-on the move towards ophthalmological research. Eye (Lond). 28, 367-380. doi: 10.1038/eye.2014.19

Choi, S., and Reddy, P. (2011). HDAC inhibition and graft versus host disease. Mol. Med. 17, 404-416. doi: 10.2119/molmed.2011.00007

Cowley, M. J., Pinese, M., Kassahn, K. S., Waddell, N., Pearson, J. V., Grimmond, S. M., et al. (2012). PINA v2.0: mining interactome modules. Nucleic Acids Res. 40, D862-D865. doi: 10.1093/nar/gkr967

Dreuw, A., Radtke, S., Pflanz, S., Lippok, B. E., Heinrich, P. C., and Hermanns, H. M. (2004). Characterization of the signaling capacities of the novel gp130-like cytokine receptor. J. Biol. Chem. 279, 36112-36120. doi: 10.1074/jbc.M401122200

Gautier, L., Cope, L., Bolstad, B. M., and Irizarry, R. A. (2004). affy-analysis of Affymetrix GeneChip data at the probe level. Bioinformatics 20, 307-315. doi: 10.1093/bioinformatics/btg405

Gene Ontology Consortium (2015). Gene ontology consortium: going forward. Nucleic Acids Res. 43, D1049-D1056. doi: 10.1093/nar/gku1179

Gentleman, R. C., Carey, V. J., Bates, D. M., Bolstad, B., Dettling, M., Dudoit, S., et al. (2004). Bioconductor: open software development for computational biology and bioinformatics. Genome Biol. 5:R80. doi: 10.1186/gb-2004-5-1 $0-\mathrm{r} 80$

Gerstein, M. B., Kundaje, A., Hariharan, M., Landt, S. G., Yan, K. K., Cheng, C., et al. (2012). Architecture of the human regulatory network derived from ENCODE data. Nature 489, 91-100. doi: 10.1038/nature11245

Ghosh, A. K., and Varga, J. (2007). The transcriptional coactivator and acetyltransferase p300 in fibroblast biology and fibrosis. J. Cell. Physiol. 213, 663-671. doi: $10.1002 /$ jcp. 21162

Gorsuch, R. A., and Hyde, D. R. (2014). Regulation of muller glial dependent neuronal regeneration in the damaged adult zebrafish retina. Exp. Eye Res. 123, 131-140. doi: 10.1016/j.exer.2013.07.012

Gu, D., Beltran, W. A., Pearce-Kelling, S., Li, Z., Acland, G. M., and Aguirre, G. D. (2009). Steroids do not prevent photoreceptor degeneration in the lightexposed T4R rhodopsin mutant dog retina irrespective of AP-1 inhibition. Invest. Ophthalmol. Vis. Sci. 50, 3482-3494. doi: 10.1167/iovs.08-3111

Hadziahmetovic, M., Kumar, U., Song, Y., Grieco, S., Song, D., Li, Y., et al. (2012). Microarray analysis of murine retinal light damage reveals changes in iron regulatory, complement, and antioxidant genes in the neurosensory retina and isolated RPE. Invest. Ophthalmol. Vis. Sci. 53, 5231-5241. doi: 10.1167/iovs.12-10204

Heinrich, P. C., Behrmann, I., Haan, S., Hermanns, H. M., Müller-Newen, G., and Schaper, F. (2003). Principles of interleukin (IL)-6-type cytokine signalling and its regulation. Biochem. J. 374, 1-20. doi: 10.1042/bj20030407

Hermanns, H. M., Radtke, S., Haan, C., Schmitz-Van de Leur, H., Tavernier, J., Heinrich, P. C., et al. (1999). Contributions of leukemia inhibitory factor receptor and oncostatin $M$ receptor to signal transduction in heterodimeric complexes with glycoprotein 130. J. Immunol. 163, 6651-6658.

Hong, S., and Song, M. R. (2014). STAT3 but not STAT1 is required for astrocyte differentiation. PLoS ONE 9:e86851. doi: 10.1371/journal.pone.0086851

Hou, T., Ray, S., Lee, C., and Brasier, A. R. (2008). The STAT3 NH2-terminal domain stabilizes enhanceosome assembly by interacting with the p300 bromodomain. J. Biol. Chem. 283, 30725-30734. doi: 10.1074/jbc.M805941200

Human Treatment and Management of Animals Act (2014). Ministry of the Environment Japan, 2014, The Law for the Humane Treatment and Management of Animals, Law No. 105 (October 1, 1973, revised May 30, 2014).

Janky, R., Verfaillie, A., Imrichová, H., Van de Sande, B., Standaert, L., Christiaens, V., et al. (2014). iRegulon: from a gene list to a gene regulatory network using large motif and track collections. PLoS Comput. Biol. 10:e1003731. doi: 10.1371/journal.pcbi.1003731

Jensen, L. J., Kuhn, M., Stark, M., Chaffron, S., Creevey, C., Muller, J., et al. (2009). STRING 8-a global view on proteins and their functional interactions in 630 organisms. Nucleic Acids Res. 37, D412-D416. doi: 10.1093/nar/ gkn760

Jiang, K., Wright, K. L., Zhu, P., Szego, M. J., Bramall, A. N., Hauswirth, W. W., et al. (2014). STAT3 promotes survival of mutant photoreceptors in inherited photoreceptor degeneration models. Proc. Natl. Acad. Sci. U.S.A. 111, E5716E5723. doi: 10.1073/pnas.1411248112

Jung, J. E., Kim, G. S., and Chan, P. H. (2011). Neuroprotection by interleukin6 is mediated by signal transducer and activator of transcription 3 and antioxidative signaling in ischemic stroke. Stroke 42, 3574-3579. doi: 10.1161/STROKEAHA.111.626648 
Kassen, S. C., Thummel, R., Campochiaro, L. A., Harding, M. J., Bennett, N. A., and Hyde, D. R. (2009). CNTF induces photoreceptor neuroprotection and Muller glial cell proliferation through two different signaling pathways in the adult zebrafish retina. Exp. Eye Res. 88, 1051-1064. doi: 10.1016/j.exer.2009.01.007

Kelsh, R. N., Brand, M., Jiang, Y. J., Heisenberg, C. P., Lin, S., Haffter, P., et al. (1996). Zebrafish pigmentation mutations and the processes of neural crest development. Development 123, 369-389.

Kisseleva, T., Bhattacharya, S., Braunstein, J., and Schindler, C. W. (2002). Signaling through the JAK/STAT pathway, recent advances and future challenges. Gene 285, 1-24. doi: 10.1016/S0378-1119(02)00398-0

Kolomeyer, A. M., and Zarbin, M. A. (2014). Trophic factors in the pathogenesis and therapy for retinal degenerative diseases. Surv. Ophthalmol. 59, 134-165. doi: 10.1016/j.survophthal.2013.09.004

Kotla, S., and Rao, G. N. (2015). Reactive oxygen species (ROS) mediate p300-dependent STAT1 protein interaction with peroxisome proliferatoractivated receptor (PPAR)-gamma in CD36 protein expression and foam cell formation. J. Biol. Chem. 290, 30306-30320. doi: 10.1074/jbc.M115. 686865

Lenkowski, J. R., and Raymond, P. A. (2014). Muller glia: stem cells for generation and regeneration of retinal neurons in teleost fish. Prog. Retin. Eye Res. 40, 94-123. doi: 10.1016/j.preteyeres.2013.12.007

Meyers, J. R., Hu, L., Moses, A., Kaboli, K., Papandrea, A., and Raymond, P. A. (2012). beta-catenin/Wnt signaling controls progenitor fate in the developing and regenerating zebrafish retina. Neural Dev. 7:30. doi: 10.1186/1749-810 4-7-30

Mitchell, A., Chang, H. Y., Daugherty, L., Fraser, M., Hunter, S., Lopez, R., et al. (2015). The InterPro protein families database: the classification resource after 15 years. Nucleic Acids Res. 43, D213-D221. doi: 10.1093/nar/g ku1243

Nakashima, K., Yanagisawa, M., Arakawa, H., Kimura, N., Hisatsune, T., Kawabata, M., et al. (1999). Synergistic signaling in fetal brain by STAT3-Smad1 complex bridged by p300. Science 284, 479-482. doi: 10.1126/science.284.54 13.479

Natoli, R., Zhu, Y., Valter, K., Bisti, S., Eells, J., and Stone, J. (2010). Gene and noncoding RNA regulation underlying photoreceptor protection: microarray study of dietary antioxidant saffron and photobiomodulation in rat retina. Mol. Vis. 16, 1801-1822.

Ni, J., Shen, Y., Wang, Z., Shao, D. C., Liu, J., Kong, Y. L., et al. (2014). P300dependent STAT3 acetylation is necessary for angiotensin II-induced profibrotic responses in renal tubular epithelial cells. Acta Pharmacol. Sin. 35, 1157-1166. doi: 10.1038/aps.2014.54

Nie, Y., Erion, D. M., Yuan, Z., Dietrich, M., Shulman, G. I., Horvath, T. L., et al. (2009). STAT3 inhibition of gluconeogenesis is downregulated by SirT1. Nat. Cell Biol. 11, 492-500. doi: 10.1038/ncb1857

Nishimura, D. (2001). BioCarta. Biotech Softw. Int. Rep. 2, 117-120. doi: $10.1089 / 152791601750294344$

Nishimura, Y., Inoue, A., Sasagawa, S., Koiwa, J., Kawaguchi, K., Kawase, R., et al. (2016). Using zebrafish in systems toxicology for developmental toxicity testing. Congenit. Anom. (Kyoto) 56, 18-27. doi: 10.1111/cga. 12142

Nishimura, Y., Martin, C. L., Vazquez-Lopez, A., Spence, S. J., AlvarezRetuerto, A. I., Sigman, M., et al. (2007). Genome-wide expression profiling of lymphoblastoid cell lines distinguishes different forms of autism and reveals shared pathways. Hum. Mol. Genet. 16, 1682-1698. doi: 10.1093/hmg/ ddm116

Nishimura, Y., Murakami, S., Ashikawa, Y., Sasagawa, S., Umemoto, N., Shimada, Y., et al. (2015a). Zebrafish as a systems toxicology model for developmental neurotoxicity testing. Congenit. Anom. (Kyoto) 55, 1-16. doi: $10.1111 /$ cga. 12079

Nishimura, Y., Sasagawa, S., Ariyoshi, M., Ichikawa, S., Shimada, Y., Kawaguchi, K., et al. (2015b). Systems pharmacology of adiposity reveals inhibition of EP300 as a common therapeutic mechanism of caloric restriction and resveratrol for obesity. Front. Pharmacol. 6:199. doi: 10.3389/fphar.2015. 00199

Nishimura, Y., Yata, K., Nomoto, T., Ogiwara, T., Watanabe, K., Shintou, T., et al. (2013). Identification of a novel indoline derivative for in vivo fluorescent imaging of blood-brain barrier disruption in animal models. ACS Chem. Neurosci. 4, 1183-1193. doi: 10.1021/cn400010t
O'Brien, C. A., and Manolagas, S. C. (1997). Isolation and characterization of the human gp130 promoter. Regulation by STATS. J. Biol. Chem. 272, 15003-15010. doi: 10.1074/jbc.272.23.15003

Oka, T., Nishimura, Y., Zang, L., Hirano, M., Shimada, Y., Wang, Z., et al. (2010). Diet-induced obesity in zebrafish shares common pathophysiological pathways with mammalian obesity. BMC Physiol. 10:21. doi: 10.1186/1472-679 3-10-21

Organisciak, D. T., and Vaughan, D. K. (2010). Retinal light damage: mechanisms and protection. Prog. Retin. Eye Res. 29, 113-134. doi: 10.1016/j.preteyeres.2009.11.004

Rattner, A., and Nathans, J. (2005). The genomic response to retinal disease and injury: evidence for endothelin signaling from photoreceptors to glia. J. Neurosci. 25, 4540-4549. doi: 10.1523/JNEUROSCI.0492-05.2005

Reich, N. C. (2013). STATs get their move on. JAKSTAT 2:e27080. doi: $10.4161 /$ jkst. 27080

Remé, C. E., Grimm, C., Hafezi, F., Marti, A., and Wenzel, A. (1998). Apoptotic cell death in retinal degenerations. Prog. Retin. Eye Res. 17, 443-464. doi: 10.1016/S1350-9462(98)00009-3

Rhee, K. D., Nusinowitz, S., Chao, K., Yu, F., Bok, D., and Yang, X. J. (2013). CNTF-mediated protection of photoreceptors requires initial activation of the cytokine receptor gp130 in Muller glial cells. Proc. Natl. Acad. Sci. U.S.A. 110, E4520-E4529. doi: 10.1073/pnas.1303604110

Samardzija, M., Wenzel, A., Aufenberg, S., Thiersch, M., Remé, C., and Grimm, C. (2006). Differential role of Jak-STAT signaling in retinal degenerations. FASEB J. 20, 2411-2413. doi: 10.1096/fj.06-5895fje

Sancho-Pelluz, J., Alavi, M. V., Sahaboglu, A., Kustermann, S., Farinelli, P., Azadi, S., et al. (2010). Excessive HDAC activation is critical for neurodegeneration in the rd1 mouse. Cell Death Dis. 1, e24. doi: 10.1038/cddis.2010.4

Sasagawa, S., Nishimura, Y., Kon, T., Yamanaka, Y., Murakami, S., Ashikawa, Y., et al. (2016). DNA damage reesponse Is involved in the developmental toxicity of mebendazole in zebrafish retina. Front. Pharmacol. 7:57. doi: 10.3389/fphar.2016.00057

Science Council of Japan (2006). Guidelines for Proper Conduct of Animal Experiments. Available online at: www.scj.go.jp/ja/ info/kohyo/pdf/kohyo-20-k16-2e.pdf

Shannon, P., Markiel, A., Ozier, O., Baliga, N. S., Wang, J. T., Ramage, D., et al. (2003). Cytoscape: a software environment for integrated models of biomolecular interaction networks. Genome Res. 13, 2498-2504. doi $10.1101 /$ gr. 1239303

Shimazawa, M., Sugitani, S., Inoue, Y., Tsuruma, K., and Hara, H. (2015). Effect of a sigma-1 receptor agonist, cutamesine dihydrochloride (SA4503), on photoreceptor cell death against light-induced damage. Exp. Eye Res. 132, 64-72. doi: 10.1016/j.exer.2015.01.017

Standards Relating to the Care and Management of Laboratory Animals and Relief of Pain (2013). Ministry of the Environment Japan, Standards Relating to the Care and Management of Laboratory Animals and Relief of Pain, 2013, Notice No. 88 (April 28, 2006, revised August 30, 2013).

Traber, K. E., Hilliard, K. L., Allen, E., Wasserman, G. A., Yamamoto, K., Jones, M. R., et al. (2015). Induction of STAT3-dependent CXCL5 expression and neutrophil recruitment by oncostatin-M during pneumonia. Am. J. Respir. Cell Mol. Biol. 53, 479-488. doi: 10.1165/rcmb.2014-0342OC

Tsuruma, K., Yamauchi, M., Sugitani, S., Otsuka, T., Ohno, Y., Nagahara, Y., et al. (2014). Progranulin, a major secreted protein of mouse adipose-derived stem cells, inhibits light-induced retinal degeneration. Stem Cells Transl. Med. 3, 42-53. doi: 10.5966/sctm.2013-0020

Ueki, Y., Le, Y. Z., Chollangi, S., Muller, W., and Ash, J. D. (2009). Preconditioninginduced protection of photoreceptors requires activation of the signaltransducing receptor gp130 in photoreceptors. Proc. Natl. Acad. Sci. U.S.A. 106, 21389-21394. doi: 10.1073/pnas.0906156106

UniProtConsortium (2015). UniProt: a hub for protein information. Nucleic Acids Res. 43, D204-D212. doi: 10.1093/nar/gku989

Watanabe, K., Nishimura, Y., Nomoto, T., Umemoto, N., Zhang, Z., Zhang, B., et al. (2012). In vivo assessment of the permeability of the blood-brain barrier and blood-retinal barrier to fluorescent indoline derivatives in zebrafish. $B M C$ Neurosci. 13:101. doi: 10.1186/1471-2202-13-101

Wen, Z., Zhong, Z., and Darnell, J. E. Jr. (1995). Maximal activation of transcription by Stat1 and Stat3 requires both tyrosine and serine phosphorylation. Cell 82, 241-250. doi: 10.1016/0092-8674(95)90311-9 
Wenzel, A., Grimm, C., Samardzija, M., and Remé, C. E. (2005). Molecular mechanisms of light-induced photoreceptor apoptosis and neuroprotection for retinal degeneration. Prog. Retin. Eye Res. 24, 275-306. doi: 10.1016/j.preteyeres.2004.08.002

Wenzel, A., Grimm, C., Seeliger, M. W., Jaissle, G., Hafezi, F., Kretschmer, R., et al. (2001). Prevention of photoreceptor apoptosis by activation of the glucocorticoid receptor. Invest. Ophthalmol. Vis. Sci. 42, 1653-1659.

Westerfield, M. (2007). A Guide for the Laboratory Use of Zebrafish (Danio rerio). Eugene: University of Oregon Press.

Winterhalter, C., Widera, P., and Krasnogor, N. (2014). JEPETTO: a Cytoscape plugin for gene set enrichment and topological analysis based on interaction networks. Bioinformatics 30, 1029-1030. doi: 10.1093/bioinformatics/ btt732

Yin, J., Brocher, J., Linder, B., Hirmer, A., Sundaramurthi, H., Fischer, U., et al. (2012). The 1D4 antibody labels outer segments of long double cone but not rod photoreceptors in zebrafish. Invest. Ophthalmol. Vis. Sci. 53, 4943-4951. doi: 10.1167/iovs.12-9511

Yuan, Z. L., Guan, Y. J., Chatterjee, D., and Chin, Y. E. (2005). Stat3 dimerization regulated by reversible acetylation of a single lysine residue. Science 307, 269-273. doi: 10.1126/science. 1105166
Yurco, P., and Cameron, D. A. (2005). Responses of Muller glia to retinal injury in adult zebrafish. Vision Res. 45, 991-1002. doi: 10.1016/j.visres.2004.10.022

Zhang, H., Dai, X., Qi, Y., He, Y., Du, W., and Pang, J. J. (2015). Histone deacetylases inhibitors in the treatment of retinal degenerative diseases: overview and perspectives. J. Ophthalmol. 2015:250812. doi: $10.1155 / 2015 / 250812$

Zhuang, S. (2013). Regulation of STAT signaling by acetylation. Cell. Signal. 25 , 1924-1931. doi: 10.1016/j.cellsig.2013.05.007

Conflict of Interest Statement: The authors declare that the research was conducted in the absence of any commercial or financial relationships that could be construed as a potential conflict of interest.

Copyright (c) 2016 Kawase, Nishimura, Ashikawa, Sasagawa, Murakami, Yuge, Okabe, Kawaguchi, Yamamoto, Moriyuki, Yamane, Tsuruma, Shimazawa, Hara and Tanaka. This is an open-access article distributed under the terms of the Creative Commons Attribution License (CC BY). The use, distribution or reproduction in other forums is permitted, provided the original author(s) or licensor are credited and that the original publication in this journal is cited, in accordance with accepted academic practice. No use, distribution or reproduction is permitted which does not comply with these terms. 